\title{
The incidence of postoperative radiotherapy- induced acute dermatitis in breast cancer and its influencing factors for Chinese women
}

This article was published in the following Dove Press journal:

OncoTargets and Therapy

\author{
Jingxian Ding ${ }^{1,2, *}$ \\ Yonghong Guo 3 ,* \\ Qingge $\mathrm{Li}^{\prime}$ \\ Jun Chen ${ }^{4}$ \\ Pinghua $\mathrm{Hu}^{4}$ \\ Qiuming Liu $^{4}$ \\ Yali $\mathrm{CaO}^{4}$ \\ Jiong $\mathrm{Wu}^{5,6}$
}

'Department of Radiation Oncology, The Third Hospital of Nanchang, Nanchang, China; '2Department of Oncology, Fudan University Shanghai Cancer Center, Shanghai, China; ${ }^{3}$ Department of Radiation Oncology, The Fourth Affiliated Hospital of Nanchang University, Nanchang, China; ${ }^{4}$ Department of Breast Surgery, The Third Hospital of Nanchang, Nanchang, China; ${ }^{5}$ Department of Breast Surgery, Fudan University Shanghai Cancer Center, Shanghai, China; ${ }^{6}$ Department of Oncology, Shanghai Medical College, Fudan University, Shanghai, China

*These authors contributed equally to this work

\begin{abstract}
Purpose: To investigate the incidence of skin acute reaction and its influencing factors in postoperative breast cancer radiotherapy patients.

Methods: One hundred and seventy three cases of breast cancer patients treated with postoperative radiotherapy were consecutively enrolled from June 1, 2016 to July 31, 2017 in our breast cancer center. Irradiation technology includes conformal intensity-modulated radiotherapy and a conventional two-dimensional one with conventional fraction. Any acute radiation dermatitis was recorded and the influencing factors were analyzed at the end of the radiation treatment.

Results: Radiotherapy-induced acute dermatitis in patients with breast-conserving surgery was relatively mild. Among the 173 patients, 33 cases had no obvious changes in the skin (grade 0); 121 cases had grade 1 skin reactions, manifested as local dark erythema and dry peeling; 29 cases had grade 2 skin reactions, characterized by edema, hyperemia, or erosion part; no grade 3 cases of skin reactions were observed. The incidence of grade 0 , grade 1 , and grade 2 reactions in all patients was $19.1 \%, 69.9 \%$, and $11.0 \%$, respectively. The severity of skin acute reaction is independent of the tumor sites, molecular subtypes, patients' age, and irradiation modalities, but it depends on the surgical types, fields treated, and planned total radiation. There is a trend favoring no chemotherapy over chemotherapy, though $p$-value is 0.074 .

Conclusion: Skin acute reaction in postoperative radiotherapy breast cancer patients is generally common but mild, and there are quite a few patients who need interruption or cessation of the radiotherapy process. The patients need to be well informed and made aware that any skin reaction will likely be mild, especially for the breast-conserving patients.
\end{abstract}

Keywords: breast neoplasm, radiotherapy, skin acute reaction, intensity modulated radiotherapy (IMRT)

\section{Introduction}

Breast cancer is still one of the most common malignant tumors and the leading cause of cancer-related death among women worldwide, including China. ${ }^{1-3}$ It is mainly treated by surgery, chemotherapy, radiotherapy, targeted therapy, and endocrine therapy. In the patients with operable breast cancer treated with standardized therapies, the prognosis is good, with the 5-year overall survival rate (OS) being over $90 \%{ }^{4,5}$

Radiotherapy has been used in the treatment of many cancer types such as breast cancer. Postoperative radiation therapy in breast cancer not only improves OS after breast-conserving surgery, but also after mastectomy in node-positive disease. . $^{6}$ Approximately, half of the patients with breast cancer receive postoperative external beam radiotherapy. However, radiotherapy itself also inevitably involves some unwanted irradiation adverse events to normal tissues, including the skin, such as 
acute dermatitis, subacute complications, and undesirable cosmetic changes. Radiation-induced dermatitis is the most common adverse effect of external radiotherapy. Severe cases necessitate interruption of radiotherapy for intolerable moist desquamation until the injury heals. However, the incidence and severity of radiation-induced dermatitis vary significantly in previous reports. It may be influenced by extrinsic factors, such as radiation dose, target volume, fraction size, and radiation technique, and also by intrinsic factors such as genetic background, breast size and geometry, age, and molecular subtypes. ${ }^{8}$ There is no clear evidence indicating that a specific product can prevent or treat radiation-induced dermatitis. $^{9}$

Here, we report the incidence of acute skin reaction and analyze its influencing factors at the end of postoperative radiotherapy for breast cancer patients in our center.

\section{Materials and methods}

\section{Patient selection}

We conducted an Institution Review Board (The Third Hospital of Nanchang Medical Ethics Committee)-approved observational study for patients undergoing postoperative radiotherapy for breast cancer from June 2016 to August 2017 in the Department of Radiation Oncology at the Third Hospital of Nanchang and the Fourth Affiliated Hospital of Nanchang University. All patients provided written informed consent form before enrollment in the study. The patients eligible for enrollment were subjects with histologic proof of a primary invasive breast carcinoma or ductal carcinoma in situ (DCIS) who underwent a planned course of continuous, definitive, or adjuvant external beam radiation therapy to the whole breast for lumpectomy or to the chest wall for mastectomy. Treatment of the regional lymph nodes, including supraclavicular and internal mammary lymph nodes, was permitted as indicated. Patients with bilateral breast cancer, or previous radiation to the same sites being treated, were excluded. All clinicopathologic and treatment planning data were retrospectively extracted from the electronic medical record. One hundred seventy six cases of radiation therapy for breast cancer patients were collected in the preset period, all of whom were female, and three patients were excluded. There were 98 cases of left breast cancer and 75 cases of right breast cancer; 34 cases of breast-conserving surgery, including six cases with lymph node metastasis and three cases of DCIS; 139 cases of modified radical mastectomy, three cases of local recurrence, and no previous radiotherapy history. The baseline clinicopathologic characteristics of patients are shown in Table 1.
Table I Patients clinicopathologic characteristics

\begin{tabular}{|c|c|c|}
\hline Characteristics & $\mathbf{N}$ & $\%$ \\
\hline \multicolumn{3}{|l|}{ Tumor sites } \\
\hline Left breast cancer & 98 & 56.6 \\
\hline Right breast cancer & 75 & 43.4 \\
\hline \multicolumn{3}{|l|}{ Molecular subtypes } \\
\hline Luminal A & 27 & 15.6 \\
\hline Luminal B & 83 & 48.0 \\
\hline HER-2 enriched & 35 & 20.2 \\
\hline Triple negative & 28 & 16.2 \\
\hline \multicolumn{3}{|l|}{ Age, years } \\
\hline$<40$ & 24 & 13.9 \\
\hline $40-50$ & 56 & 32.4 \\
\hline $50-60$ & 66 & 38.2 \\
\hline$>60$ & 27 & 15.6 \\
\hline \multicolumn{3}{|l|}{ Types of surgery } \\
\hline Lumpectomy & 34 & 19.7 \\
\hline Mastectomy & 139 & 80.3 \\
\hline \multicolumn{3}{|l|}{ Chemotherapy } \\
\hline Yes & 157 & 90.8 \\
\hline No & 16 & 9.2 \\
\hline \multicolumn{3}{|l|}{ Radiation methods } \\
\hline IMRT & 164 & 94.8 \\
\hline $2 \mathrm{D}$ & 9 & 5.2 \\
\hline \multicolumn{3}{|l|}{ Fields treated } \\
\hline Whole breast & 28 & 16.2 \\
\hline Whole breast+SCN & 6 & 3.5 \\
\hline Chest wall+SCN & 111 & 64.2 \\
\hline Chest wall+SCN+IMN & 29 & 16.8 \\
\hline \multicolumn{3}{|l|}{ Planned total radiation (Gy) } \\
\hline 50 & 139 & 80.3 \\
\hline 60 & 30 & 17.3 \\
\hline$>60$ & 4 & 2.3 \\
\hline
\end{tabular}

Abbreviations: 2D, two-dimensional; IMN, internal mammary nodes; IMRT, intensity-modulated radiotherapy; SCN, supraclavicular nodes.

\section{Radiation modalities}

The radiation oncologists defined the target volume, and the radiation physicists made the dose distribution, which was confirmed by the radiation oncologists. Planning target volume was contoured under radiation therapy oncology group (RTOG) guidelines for intensity-modulated radiotherapy (IMRT) patients. All radiation planning was done in Pinnacle (Philips Medical Systems). Radiation plans were designed using IMRT. IMRT involved tangential sliding window continuous segmentation with inverse planning of an optimization structure derived from the target volume. Conventional two-dimensional (2D) radiation usually had three fields, one for supraclavicular lymph nodes and the other two for chest wall (Figure 1). Patients treated with conventional 2D irradiation were normally radiated with 7-8 MeV electron in chest wall field, while 9-12 MeV electron and photon alternately mixed in supraclavicular nodes field. Selection between conventional 2D irradiation 

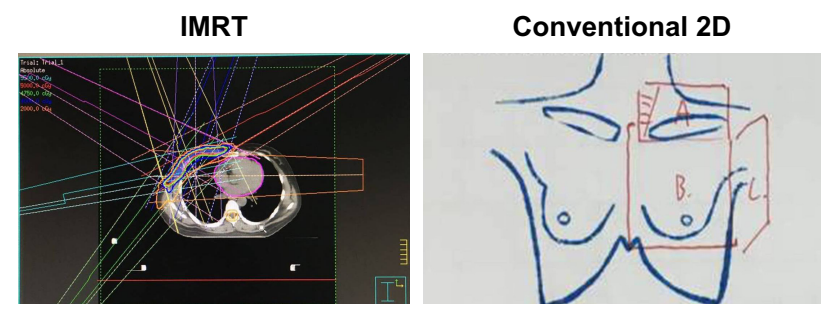

Figure I Image of radiation modalities.

Notes: IMRT involved tangential sliding window continuous segmentation with inverse planning of an optimization structure derived from the target volume. Conventional 2D radiation usually had three fields, field $A$ for supraclavicular lymph nodes, field B for medial side of chest wall, and field $C$ for lateral side of chest wall. Abbreviations: 2D, two-dimensional; IMRT, intensity-modulated radiotherapy.

and IMRT was at patients' discretion, with bias toward conventional 2D irradiation in some cases of poor, but no patients with lumpectomy or $>4$ involved lymph nodes were irradiated with conventional 2D for safety consideration. All patients were prescribed with a total radiation dose of $50 \mathrm{~Gy}$ in 25 daily fractions. A boost field of an additional 10-16 Gy was applied to the tumor bed using electrons. The physician determined the dose and the boost field design for tumor bed. Conventional fractions were treated to all patients at $200 \mathrm{cGy}$ daily fractions to $5,000 \mathrm{cGy}$ for postmastectomy adjuvant radiotherapy or to 6,000-6,600 cGy for lumpectomy or local recurrence disease, respectively. Internal mammary nodes were generally only treated if clinically involved, typically using IMRT. When lumpectomy tumor bed boost was used, it was administered in $200 \mathrm{cGy}$ fractions to a total dose to $1,000-1,600$ cGy with electron, while in patients with DCIS, no boost.

\section{Education prior to radiation}

We implement routine health education on all patients before radiation therapy, guide the patients to expose irradiation sites and avoid friction with appropriate cotton underwear, not to expose the irradiation sites to skin irritation detergent, avoid scratching and using alcoholic disinfectant, have diet with high protein, high vitamin and easy-to-digest food, eat more fresh fruits and vegetables, and not to eat spicy food and drink hot water, and some patients use Biafine or other skin creams on their own will. Previous reports showed that there was no overall difference between best supportive care and use of Biafine in the prevention of, time to, or duration of radiation-induced dermatitis. ${ }^{8}$

\section{Assessment of acute skin toxicity}

Toxicity grading was in accordance with the RTOG scoring criteria for acute radiation dermatitis using physicianreported outcomes and scored as the most severe toxicity observed. This scoring system provides a ranking of skin reaction from no signs of dermatitis (grade 0 ), to severe and ulcerating dermatitis (grade 4) (Table 2). ${ }^{10}$ Dermatitis assessments were performed at the end of radiotherapy. When grade 1 or mild grade 2 skin reactions occur, the radiotherapy can be continued, and the radiation field should be kept clean and dry, avoiding the friction stimulation of the hard clothes. Once the confluent wet desquamation occurred, the radiotherapy was terminated, and for the potential infected patients, 3 days of oral antibiotics were necessary. Additionally, after completion of radiotherapy, the patients completed a patient questionnaire booklet for the 2 weeks immediately after radiotherapy completion.

\section{Statistical analysis}

The primary endpoints of the study were the frequency of postoperative radiotherapy-induced acute dermatitis in breast cancer and its influencing factors assessed by the physicians under the RTOG scoring criteria for acute radiation dermatitis. The maximal grade of this adverse event during treatment was recorded for each patient. Using a single-factor analysis of variance and the $\chi^{2}$-test to compare between each potential influencing factor by SPSS 15.0 statistical software, $p<0.05$ was statistically significant.

\section{Results}

A total of 176 patients with radiation therapy for breast cancer were enrolled between June 2016 and August 2017. All irradiation sites were recorded and assessed at the completion of scheduled prescription dose, and then we scheduled a follow-up at 2 weeks after irradiation completion. Of the 176 patients, there were three patients who did not complete the planned radiotherapy, one for ischemic cardiac disease potentially caused by anthracycline-based chemotherapy, and

Table 2 Radiation therapy oncology group scoring criteria for acute radiation dermatitis

\begin{tabular}{|c|c|c|c|c|c|}
\hline & Grade 0 & Grade I & Grade 2 & Grade 3 & Grade 4 \\
\hline Skin & $\begin{array}{l}\text { No change } \\
\text { over baseline }\end{array}$ & $\begin{array}{l}\text { Follicular, faint, or dull } \\
\text { erythema/epilation/ } \\
\text { dry desquamation/ } \\
\text { decreased sweating }\end{array}$ & $\begin{array}{l}\text { Tender or bright } \\
\text { erythema, patchy } \\
\text { moist desquamation/ } \\
\text { moderate edema }\end{array}$ & $\begin{array}{l}\text { Confluent, moist } \\
\text { desquamation } \\
\text { other than skin } \\
\text { folds, pitting edema }\end{array}$ & $\begin{array}{l}\text { Ulceration, } \\
\text { hemorrhage, } \\
\text { necrosis }\end{array}$ \\
\hline
\end{tabular}


the other two for serious anxiety. Consequently, 173 patients were eligible for evaluation of the primary endpoint. Overall, among the patients qualified, at the last fraction of radiation, there were no obvious skin change over baseline in 33 cases; 121 subjects with grade 1 skin reactions manifested as local follicular, faint, or dull erythema/epilation/dry desquamation/ decreased sweating; 19 patients suffered from grade 2 skin reactions, characterized by tender or bright erythema, patchy moist desquamation/moderate edema (Table 3). No skin reactions of were found in this study (Figure 2). Grade 1 skin reactions normally occurred after the skin dosage up to $>3,000 \mathrm{cGy}$, and grade 2 skin reactions usually appeared after the skin dosage reached $>4,000 \mathrm{cGy}$. All of the

Table 3 Maximal radiation dermatitis grade related to the clinicopathologic characteristics, assessed by radiation therapy oncology group score

\begin{tabular}{|c|c|c|c|c|c|c|c|}
\hline \multirow[t]{2}{*}{ Characteristics } & \multicolumn{2}{|c|}{ Grade 0} & \multicolumn{2}{|c|}{ Grade I } & \multicolumn{2}{|c|}{ Grade 2} & \multirow[t]{2}{*}{$p$-value } \\
\hline & $\mathbf{N}$ & $\%$ & $\mathbf{N}$ & $\%$ & $\mathbf{N}$ & $\%$ & \\
\hline \multicolumn{8}{|l|}{ Tumor sites } \\
\hline Left breast & 19 & 19.4 & 68 & 69.4 & II & 11.2 & 0.983 \\
\hline Right breast & 14 & 18.7 & 53 & 70.7 & 8 & 10.7 & \\
\hline \multicolumn{8}{|l|}{ Molecular subtypes } \\
\hline Luminal A & 9 & 33.3 & 17 & 63.0 & I & 3.7 & 0.332 \\
\hline Luminal B & 15 & 18.1 & 60 & 72.3 & 8 & 9.6 & \\
\hline HER-2 enriched & 5 & 14.3 & 24 & 68.6 & 6 & 17.1 & \\
\hline Triple negative & 4 & 14.3 & 20 & 71.4 & 4 & 14.3 & \\
\hline \multicolumn{8}{|l|}{ Age, years } \\
\hline$<40$ & 5 & 20.8 & 19 & 79.2 & 0 & 0.0 & 0.362 \\
\hline $40-50$ & 9 & 16.1 & $4 \mid$ & 73.2 & 6 & 10.7 & \\
\hline $50-60$ & 16 & 24.2 & 41 & 62.1 & 9 & 13.6 & \\
\hline$>60$ & 3 & 11.1 & 20 & 74.1 & 4 & 14.8 & \\
\hline \multicolumn{8}{|l|}{ Types of surgery } \\
\hline Lumpectomy & 18 & 52.9 & 14 & 41.2 & 2 & 5.9 & $0.000 *$ \\
\hline Mastectomy & 15 & 10.8 & 107 & 77.0 & 17 & 12.2 & \\
\hline \multicolumn{8}{|l|}{ Chemotherapy } \\
\hline Yes & 27 & 17.2 & 111 & 70.7 & 19 & 12.1 & 0.074 \\
\hline No & 6 & 37.5 & 10 & 62.5 & 0 & 0.0 & \\
\hline \multicolumn{8}{|l|}{ Radiation methods } \\
\hline IMRT & 31 & 18.9 & 115 & 70.1 & 18 & 11.0 & 0.969 \\
\hline $2 \mathrm{D}$ & 2 & 22.2 & 6 & 66.7 & 1 & 11.1 & \\
\hline \multicolumn{8}{|l|}{ Fields treated } \\
\hline Whole breast & 15 & 53.6 & 11 & 39.3 & 2 & 7.1 & $0.000 *$ \\
\hline Whole breast+SCN & 2 & 33.3 & 4 & 66.7 & 0 & 0.0 & \\
\hline Chest wall+SCN & II & 10.0 & 87 & 79.1 & 13 & 11.8 & \\
\hline Chest wall+SCN+IMN & 4 & 13.8 & 21 & 72.4 & 4 & 13.8 & \\
\hline \multicolumn{8}{|c|}{ Planned total radiation (Gy) } \\
\hline 50 & 20 & 14.4 & 105 & 75.5 & 14 & 10.1 & $0.000 *$ \\
\hline 60 & 13 & 43.3 & 14 & 46.7 & 3 & 10.0 & \\
\hline$>60$ & 0 & 0.0 & 2 & 50.0 & 2 & 50.0 & \\
\hline
\end{tabular}

Notes: Using a single-factor analysis of variance and the $\chi^{2}$-test to compare between each potential influencing factor by SPSS 15.0 statistical software. *Statistically significant.

Abbreviations: 2D, two-dimensional; IMN, internal mammary nodes; IMRT, intensity-modulated radiotherapy; SCN, supraclavicular nodes.

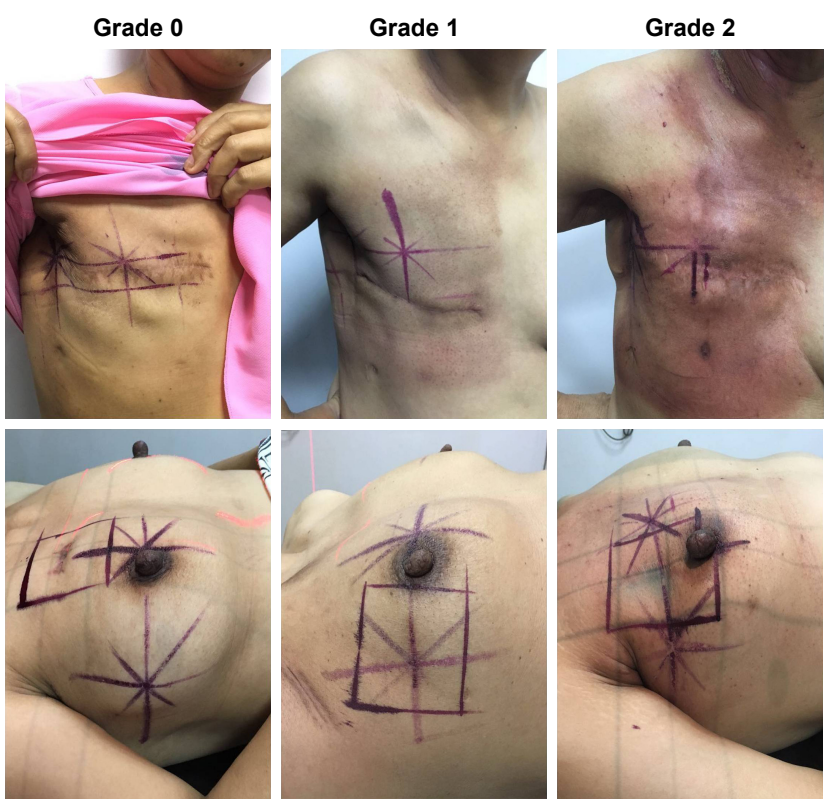

Figure 2 The representative images of grade 0 , grade 1 , and grade 2 dermatitis in postmastectomy radiotherapy (top panel) and postlumpectomy radiotherapy (bottom panel).

Notes: Grade 0: no obvious skin change over baseline; grade I: skin reactions manifested as local follicular, faint, or dull erythema/epilation/dry desquamation/ decreased sweating; grade 2: skin reactions, characterized by tender or bright erythema, patchy moist desquamation/moderate edema. All pictures were taken on the day of the last fraction.

radiation-induced skin lesions were healed within 2 weeks after completion of radiotherapy.

We further analyzed the potential influencing factors related to the severity of radiotherapy-induced skin lesions. There was no difference in the severity of skin acute reaction between left breast cancer and right breast cancer. Similarly, no difference was found in molecular subtypes, patients' age, and irradiation modalities. There is a trend favoring no chemotherapy over chemotherapy, though $p$-value is 0.074 . However, the severity of skin acute reaction does depend on the surgical types, fields treated, and planned total radiation; Table 3 shows the univariate analysis of factors as they relate to radiation dermatitis.

Patients with grade 2 acute dermatitis recovered within 2 weeks after the completion of irradiation through proper local care, leaving radiation field skin pigmentation, most of which did not have a substantial impact on the process of radiotherapy.

\section{Discussion}

Radiotherapy plays an important role in reducing local recurrence by about two-thirds and even metastasis, improving disease-free survival and prolonging the OS. ${ }^{11}$ With improving OS rates in breast cancer, there is an emerging focus 
on the quality of life after adjuvant treatment. However, irradiation is often accompanied by a variety of radiation injuries. Acute radiation dermatitis is a common side effect of radiotherapy in many forms of cancer including breast cancer. The severity of the reaction may depend on the radiotherapy fraction schedules, the total dose, the treated skin area, and also individual variations. ${ }^{12}$ For short of blood supply, postoperative breast cancer chest wall skin is not well tolerated, and easy to damage in the radiation field of skin, as manifested by erythema, edema, erosion, ulcer, or even serious. Normally, the severity of this symptom may be related to the prescription dose and fractionation delivered to the skin and to patient-related factors such as obesity, smoking, and use of radiosensitizing chemotherapy. ${ }^{13-15}$ Though the exact mechanisms of acute radiation dermatitis are still unknown, it may be associated with an inflammatory cascade mediated by cytokines. ${ }^{16}$ In vitro studies showed that there were morphologic alterations to the normal histologic characteristics, including a marked decrease in basal cell proliferation, endothelial cell damage, resultant vasodilation with altered membrane permeability, and inflammatory cytokine release by irradiation. ${ }^{17,18}$ Corticosteroids may reduce the incidence and severity of radiation dermatitis and delay the onset of the maximal clinical symptoms. ${ }^{19,20}$

In our study, the results showed that the acute skin reaction of irradiation in postoperative radiotherapy breast cancer patients is generally mild and tolerable; grade 1 dermatitis accounted for about $69.9 \%$, grade 2 dermatitis accounted for about $11.0 \%$, and no grade 3 or higher dermatitis was observed in the patients studied. Nearly $19.1 \%$ of our patients showed no radiation-induced skin reaction at all. Previous reports showed that radiation-induced skin reactions were observed in $\sim 90 \%$ of breast cancer patients undergoing adjuvant postoperative radiotherapy. ${ }^{21,22}$ In our data, the incidence is lower than the previous reports. It needs to be studied further if the skin color affects it. We found that there is high individual variation in the development of radiation-induced dermatitis. There must be a number of factors, which were poorly understood to our knowledge, behind skin sensitivity to radiation and development of dermatitis. Through univariate analysis, our study suggests that the surgical methods, irradiation fields, and planned total radiation dosage may be the possible influencing factors, and there is also a trend favoring no chemotherapy over chemotherapy; however, there is no difference in the severity of skin acute reaction between sides of breast, molecular subtypes, patients' age, and irradiation modalities. Nevertheless, the radiationinduced dermatitis may affect patient's compliance with the treatment and may be one potential reason for discontinuing the use of a potentially curative treatment. ${ }^{23,24}$

In conclusion, radiotherapy is an important part in the management of postmastectomy breast cancer patients with metastatic lymph nodes and most patients with lumpectomy. The incidence of postoperative radiotherapy-induced acute dermatitis is common but generally mild in Chinese breast cancer women, and quite a few patients' radiation process will be interrupted. Consequently, breast cancer patients with radiation indications should not give up for fear of radiationinduced acute skin reactions.

\section{Acknowledgments}

The authors alone are responsible for the content and writing of the paper. The study was sponsored by the Natural Science Foundation of Jiangxi province (No 20171BAB205057).

\section{Disclosure}

The authors report no conflicts of interest in this work.

\section{References}

1. Chen W, Zheng R, Baade PD, et al. Cancer statistics in China, 2015. CA Cancer J Clin. 2016;66(2):115-132.

2. Siegel RL, Miller KD, Jemal A. Cancer statistics, 2017. CA Cancer J Clin. 2017;67(1):7-30.

3. Zeng H, Zheng R, Zhang S, Zou X, Chen W. Female breast cancer statistics of 2010 in China: estimates based on data from 145 populationbased cancer registries. J Thorac Dis. 2014;6(5):466-470.

4. Jung KW, Won YJ, Oh CM, Kong HJ, Lee DH, Lee KH; Community of Population-Based Regional Cancer Registries. Cancer statistics in Korea: incidence, mortality, survival, and prevalence in 2014. Cancer Res Treat. 2017;49(2):292-305.

5. DeSantis C, Ma J, Bryan L, Jemal A. Breast cancer statistics, 2013. CA Cancer J Clin. 2014;64(1):52-62.

6. McGale P, Taylor C, Correa C, et al; EBCTCG (Early Breast Cancer Trialists' Collaborative Group). Effect of radiotherapy after mastectomy and axillary surgery on 10-year recurrence and 20-year breast cancer mortality: meta-analysis of individual patient data for 8135 women in 22 randomised trials. Lancet. 2014;383(9935):2127-2135.

7. Darby S, McGale P, Correa C, et al; Early Breast Cancer Trialists' Collaborative Group (EBCTCG). Effect of radiotherapy after breastconserving surgery on 10-year recurrence and 15-year breast cancer death: meta-analysis of individual patient data for 10,801 women in 17 randomised trials. Lancet. 2011;378(9804):1707-1716.

8. Fisher J, Scott C, Stevens R, et al. Randomized phase III study comparing best supportive care to Biafine as a prophylactic agent for radiationinduced skin toxicity for women undergoing breast irradiation: radiation therapy oncology group (RTOG) 97-13. Int J Radiat Oncol Biol Phys. 2000;48(5):1307-1310.

9. Aysan E, Idiz UO, Elmas L, Saglam EK, Akgun Z, Yucel SB. Effects of boron-based gel on radiation-induced dermatitis in breast cancer: a double-blind, placebo-controlled trial. J Invest Surg. 2017;30(3): 187-192.

10. Cox JD, Stetz J, Pajak TF. Toxicity criteria of the Radiation Therapy Oncology Group (RTOG) and the European Organization for Research and Treatment of Cancer (EORTC). Int J Radiat Oncol Biol Phys. 1995; 31(5):1341-1346.

11. Dewar JA. Postmastectomy radiotherapy. Clin Oncol (R Coll Radiol). 2006;18(3):185-190. 
12. Wang Q, Jie W, Liang Z, Wu H, Cheng J. Postmastectomy intensity modulation radiated therapy of chest wall and regional nodes: retrospective analysis of the performance and complications up for 5 years. Medicine (Baltimore). 2017;96(39):e7956.

13. Miller RC, Schwartz DJ, Sloan JA, et al. Mometasone furoate effect on acute skin toxicity in breast cancer patients receiving radiotherapy: a phase III double-blind, randomized trial from the North Central Cancer Treatment Group N06C4. Int J Radiat Oncol Biol Phys. 2011;79(5): 1460-1466.

14. Jung K, Sabri S, Hanson J, et al. Elevated ARG1 expression in primary monocytes-derived macrophages as a predictor of radiation-induced acute skin toxicities in early breast cancer patients. Cancer Biol Ther. 2015;16(9):1281-1288

15. Chen MF, Chen WC, Lai CH, Hung CH, Liu KC, Cheng YH. Predictive factors of radiation-induced skin toxicity in breast cancer patients. BMC Cancer. 2010;10:508.

16. Lee JH, Kay CS, Maeng LS, et al. The clinical features and pathophysiology of acute radiation dermatitis in patients receiving tomotherapy. Ann Dermatol. 2009;21(4):358-363.

17. Harper JL, Franklin LE, Jenrette JM, Aguero EG. Skin toxicity during breast irradiation: pathophysiology and management. South Med J. 2004;97(10):989-993.

18. Miller RC, Schwartz DJ, Sloan JA, et al. Mometasone furoate effect on acute skin toxicity in breast cancer patients receiving radiotherapy: a phase III double-blind, randomized trial from the North Central Cancer Treatment Group N06C4. Int J Radiat Oncol Biol Phys. 2011;79(5): $1460-1466$.
19. Salvo N, Barnes E, van Draanen J, et al. Prophylaxis and management of acute radiation-induced skin reactions: a systematic review of the literature. Curr Oncol. 2010;17(4):94-112.

20. Ulff E, Maroti M, Serup J, Nilsson M, Falkmer U. Prophylactic treatment with a potent corticosteroid cream ameliorates radiodermatitis, independent of radiation schedule. Radiother Oncol. 2017;122(1):50-53.

21. Aistars J. The validity of skin care protocols followed by women with breast cancer receiving external radiation. Clin J Oncol Nurs. 2006; 10(4):487-492.

22. Aysan E, Idiz UO, Elmas L, Saglam EK, Akgun Z, Yucel SB. Effects of boron-based gel on radiation-induced dermatitis in breast cancer: a double-blind, placebo-controlled trial. J Invest Surg. 2017;30(3): 187-192.

23. Wang SL, Li YX, Zhang BN, et al. Epidemiologic study of compliance to postmastectomy radiation therapy guidelines in breast cancer patients in China between 1999 and 2008. Pract Radiat Oncol. 2013;3(3): 209-215.

24. Chu QD, Caldito G, Miller JK, Townsend B. Postmastectomy radiation for N2/N3 breast cancer: factors associated with low compliance rate. J Am Coll Surg. 2015;220(4):659-669.
OncoTargets and Therapy

\section{Publish your work in this journal}

OncoTargets and Therapy is an international, peer-reviewed, open access journal focusing on the pathological basis of all cancers, potential targets for therapy and treatment protocols employed to improve the management of cancer patients. The journal also focuses on the impact of management programs and new therapeutic agents and protocols on

\section{Dovepress}

patient perspectives such as quality of life, adherence and satisfaction The manuscript management system is completely online and includes a very quick and fair peer-review system, which is all easy to use. Visit http://www.dovepress.com/testimonials.php to read real quotes from published authors. 